\title{
Theoretical evaluation of Cox's interaction model of client health behavior for health promotion in adult women
}

\author{
Youlim Kim, Hyeonkyeong Lee, Gi Wook Ryu \\ College of Nursing, Mo-Im Kim Nursing Research Institute, Yonsei University, Seoul, Korea
}

\begin{abstract}
This study aimed to evaluate Cox's interaction model of client health behavior (IMCHB) as used in studies on women's health. Using keyword combinations of "women" and "IMCHB" or "interaction model of client health behavior," we searched the PubMed, MEDLINE, Embase, and RISS databases for studies on the promotion of women's health published from January 2009 to April 2019. Finally, 11 studies were selected and evaluated according to seven criteria for theory evaluation, which combined Fawcett's theory evaluation criteria and Chinn and Kramer's criteria. We found that the IMCHB corresponds to a verifiable practical level of a middle-range theory, although it may be partially abstract. It contains all four concepts of the metaparadigm of nursing, in terms of a holistic philosophical approach. A theoretical evaluation demonstrated that the IMCHB has significance, generality, testability, empirical adequacy, and pragmatic adequacy for nursing practice and research. However, the lack of clear conceptual definitions and the presence of complex relationships among concepts resulted in a lack of internal consistency and parsimony. According to an in-depth verification through a review of the literature, the IMCHB has been used as a health promotion intervention strategy for various populations of women and has led to useful results in nursing practice. The IMCHB was confirmed to be a suitable theory for experimental and clinical research. Future research can build on this middle-range theory for women's health research and practice.
\end{abstract}

Keywords: Health behavior; Health promotion; Nursing theory; Women

\author{
Received: May 8, 2020 \\ Revised: June 11, 2020 \\ Accepted: June 13, 2020 \\ Corresponding author: \\ Gi Wook Ryu \\ College of Nursing, Mo-Im Kim \\ Nursing Research Institute, Yonsei \\ University, 50-1 Yonsei-ro, \\ Seodaemun-gu, Seoul 03722, Korea \\ Tel: +82-2-2228-3237 \\ E-mail:smartryu14@naver.com
}

\section{Introduction}

Women, who make up half of the global population, have unique health needs from childhood to old age. The United Nations emphasized improving women's health and quality of life in its Sustainable Development Goals [1]. These agendas broadened the concept of women's social activities and gender equality, and increased the number of studies related to women's health [2]. In particular, the conceptual focus of women's health expanded from maternal health, which is focused on pregnancy and childbirth, to include sexual health, depression, drinking, and smoking among women, and is transitioning to encompass gender-centered promotion of women's health [2]. Due to these changes, researchers in the field of women's health should consider sociocultural influences, approach the holistic context of life from individuals to motherhood and beyond, and seek to understand phenomena in terms of human interactions [3].

Nursing theory is necessary as a framework for testing various phenomena in the field of nursing in the present rapidly changing social and cultural environment [4], and through such research, scientific knowledge about nursing can be accumulated. Nursing theory provides a conceptual basis for understanding the relationship between theoretical and practical nursing [5].

In order to study women's health promotion, it is necessary to

This is an Open Access article distributed under the terms of the Creative Commons Attribution License (http://creativecommons.org/licenses/by/4.0) which permits unrestricted use, distribution, and reproduction in any medium, provided the original work is properly cited.

Copyright (c) 2020 Korean Society of Women Health Nursing 


\section{Summary statement}

\section{- What is already known about this topic?}

Cox's interaction model of client health behavior (IMCHB) was previously analyzed in research articles published until 1995. Although the IMCHB has been regularly used thereafter, no recent theoretical evaluations have been conducted.

\section{- What this paper adds}

This review of 11 studies on women's health that applied the IMCHB found that the IMCHB has significance, generality, testability, empirical adequacy, and pragmatic adequacy, contributing useful results for nursing practice. However, it lacks internal consistency and parsimony due to its complex concepts and relationships.

- Implications for practice, education, and/or policy

An integrated understanding and analysis of nursing theories can guide nursing services and contribute to the advancement of the role of professional nurses.

comprehensively analyze and evaluate relevant theories by considering various aspects of the physical, mental, and social environment of the population [6]. Among the various theories related to health behavior, Cox's interaction model of client health behavior (IMCHB) includes client-professional interaction as a main concept, highlighting nurses' professional roles and their provision of patient-centered nursing in consideration of the context of the target population [7]. In addition, Cox's IMCHB is a nursing model that emphasizes the roles and interactions of clients and professionals and is used as a theoretical framework in various areas of nursing practice and research to contribute to nursing theoretical knowledge [8].

In order to properly explain phenomena encountered in nursing practice, an in-depth critical understanding of a theory is required before applying it. Evaluation of nursing theories can contribute to the consolidation of nursing epistemology and to the further development and improvement of theories [9]. A previous study evaluated the IMCHB using research papers published from 1983 to 1994, the first decade after the theory was developed [9], but no analyses and evaluations of this theory have been conducted for about 25 years thereafter. Therefore, reviewing the research related to women's health that has applied IMCHB in the last 11 years may suggest future research directions. In this study, as Cox's IMCHB is a middle-range theory, we reviewed studies that applied the IMCHB to adult women who were not hospitalized for specific diseases.

The purpose of this study was 1) to conduct a theoretical analysis and evaluation of research in the field of women's health applying Cox's IMCHB published in domestic and international research journals in the past 11 years (January 2009 to April 2019), and 2) to propose a strategy for nursing theory, research, and practice development for women's health promotion based on this analysis and evaluation.

\section{Methods}

Ethics statement: This study was exempted from the requirement to obtain informed consent because it was a literature review.

\section{Design and samples}

This study was a literature review analyzing papers on women's health that applied Cox's IMCHB and were published in domestic and international journals within the past 11 years, from January 2009 to April 2019.

\section{Data sources}

A search was conducted for studies that applied the IMCHB in relation to the health of female outpatients and were published in peer-reviewed journals in Korean or English between January 2009 and April 2019. We searched four online databases: PubMed, MEDLINE, Embase, and the Research Information Sharing Service (RISS). Terms related to the theory were identified by referring to the keywords used in theoretical evaluation papers dealing with the IMCHB [9]. We conducted a preliminary search using the terms "interaction model of client health behavior" or "Cox's interaction model" in combination with "women." The preliminary results showed that many articles used the abbreviation "IMCHB" for "interaction model of client health" throughout the text, and it was confirmed that few articles were found when "Cox" was added as a term. Therefore, we used the following final combination of keywords:

$((($ Female"[Mesh]) OR "(Women"[Mesh]))) AND (("IM- 
CHB") OR ("interaction model of client health behavior")).

\section{Study selection}

The literature selection criteria for this study were: (1) the IM$\mathrm{CHB}$ was applied, and (2) the participants were adult women. The criteria for exclusion were: (1) the IMCHB application was not clearly explained, or the participants were (2) inpatients or (3) children and adolescents.

According to the results obtained by searching the international databases using the search terms, 52 studies were found (nine in PubMed, 10 in MEDLINE, 24 in Embase, seven in RISS, and two via hand-searching). After duplicates were removed ( $\mathrm{n}=18)$, two reviewers (YK and GWR) independently screened a total of 34 articles focusing on titles and abstracts. The same reviewers then independently reviewed the full-text articles to determine whether each article was appropriate for inclusion in this analysis. One study was excluded because the original text was inaccessible and 22 articles met the exclusion criteria (6 studies did not apply the IMCHB, four studies involved inpatients, and 12 studies sampled non-adult women), so 11 studies were included in the analysis (Figure 1).

\section{Criteria for the evaluation and analysis of theory and literature}

In this study, Fawcett's theory analysis and evaluation steps were used as the main theoretical evaluation criteria [10]. Fawcett [10] presented the analysis and evaluation of theories as involving the stages of "theoretical analysis" and "theoretical evaluation." Fawcett's evaluation criteria for theories include significance, internal consistency, parsimony, testability, empirical adequacy, and pragmatic adequacy. Chinn and Kramer [11] proposed different evaluation criteria for theories, and additionally presented generality as a criterion to evaluate theories. Generality refers to assessing whether a theory can explain a wide scope of experiences and phenomena [11]. Therefore, we added the generality criterion of Chinn and Kramer [11] to Fawcett's evaluation criteria to analyze the IMCHB from various viewpoints. Consequently, in this study, the theory and literature were evaluated according to seven criteria: importance, internal consistency, parsimony, generality, testability, empirical adequacy, and practical adequacy.

\section{Results}

\section{General characteristics of the reviewed literature}

The reviewed studies included five international studies (45.5\%) and six studies from South Korea (54.5\%) (Table 1). Ten studies $(90.9 \%)$ were quantitative, and one $(9.1 \%)$ was qualitative. The participants in the studies were minority groups (immigrants or multicultural women) and low-income women in five studies $(45.5 \%)$, pregnant women in two studies $(18.2 \%)$, postpartum women in one study (9.1\%), women with gynecological cancer in one study (9.1\%), women with osteoarthritis in one study (9.1\%), and older adults in one study (9.1\%).

\section{Analysis of the theory}

\section{Scope: Level of abstraction}

The concepts of a theory play a fundamental role in its construction, and the object to which each concept is addressed should be empirically defined $[12,13]$.

Cox's IMCHB encompasses three major elements: the client background, client-professional interaction, and health outcomes of the client (Table 2). However, these concepts are not clearly explained, and there is no specific statement on how to measure them. As the IMCHB does not address specific

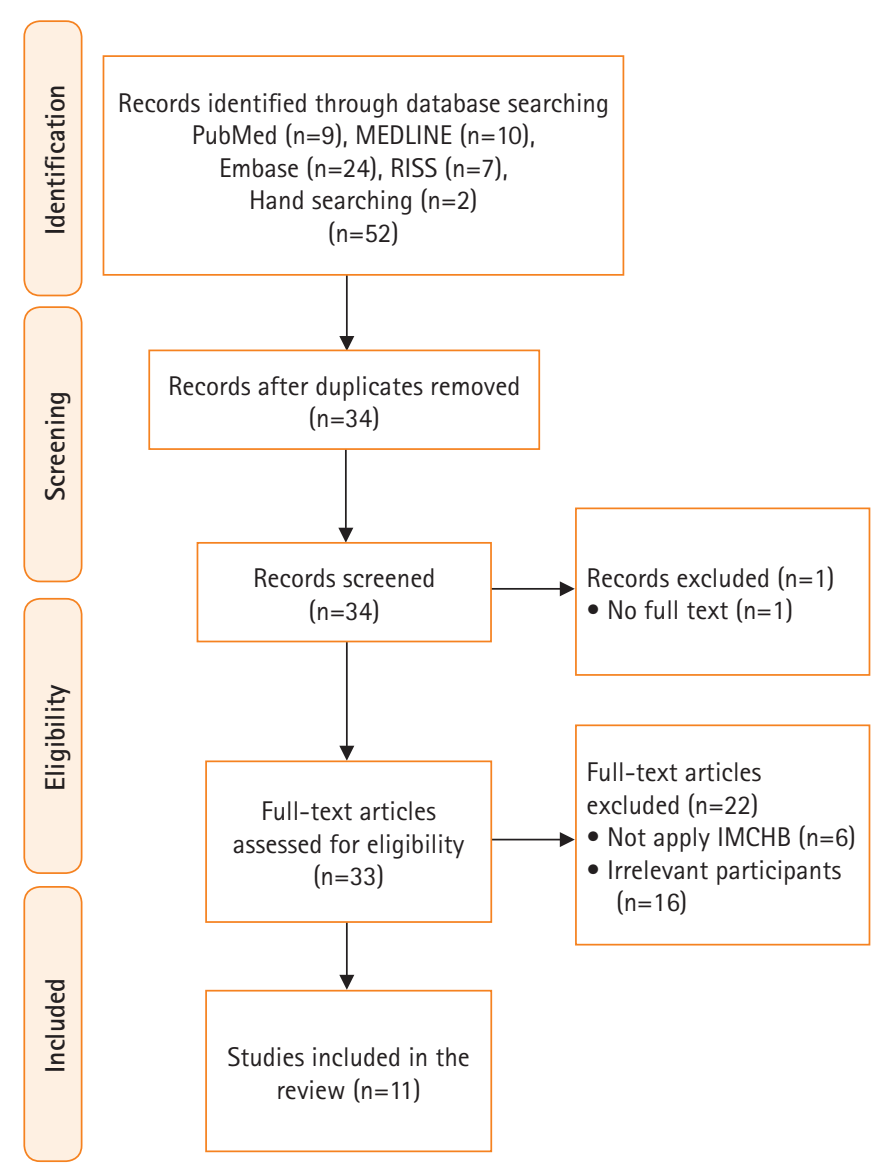

Figure 1. PRISMA flow diagram.

IMCHB: interaction model of client health behavior; RISS: Research Information Sharing Service. 
health-related situations and was designed to be applied to a wide range of health care decisions and behaviors, it is necessary to keep in mind that the concepts highlighted may vary depend-

Table 1. Characteristics of studies $(n=11)$

\begin{tabular}{llc}
\hline Variable & Categories & $\mathrm{n}(\%)$ \\
\hline Country & International & $5(45.5)$ \\
& South Korea & $6(54.5)$ \\
Research design & Quantitative & $10(90.9)$ \\
& Non-experimental design: Survey & $3(27.3)$ \\
& Experimental design: Quasi-experimental & $7(63.6)$ \\
& Qualitative & $1(9.1)$ \\
Population & Minority and low-income women & $5(45.5)$ \\
& Pregnant women & $2(18.2)$ \\
& Postpartum women & $1(9.1)$ \\
& Women with osteoarthritis & $1(9.1)$ \\
& Women with gynecological cancer & $1(9.1)$ \\
& Older adults & $1(9.1)$ \\
\hline
\end{tabular}

ing on the research question [14]. Therefore, the IMCHB corresponds to a verifiable practical level of a middle-range theory, and it may be partially abstract due to the lack of concrete descriptions of specific nursing phenomena and clients.

\section{Context: Metaparadigm concepts and propositions}

To analyze the context of the IMCHB as a theory, it is necessary to ensure that it presents a philosophical opinion based on a metaparadigm and evidence [10].

Cox [7] recognized human beings as independent entities capable of determining their own health behaviors, focused on human singularity, and identified environmental factors as personal factors, economic resources, and use of health care facilities related to background variables. In the IMCHB, "health" is described as positive behaviors obtained through interaction with experts who have recognized the characteristics of the client, and five variables are suggested to measure health outcomes (e.g., health status indicators and healthcare utilization). Nurs-

Table 2. Conceptual definition of elements and variables

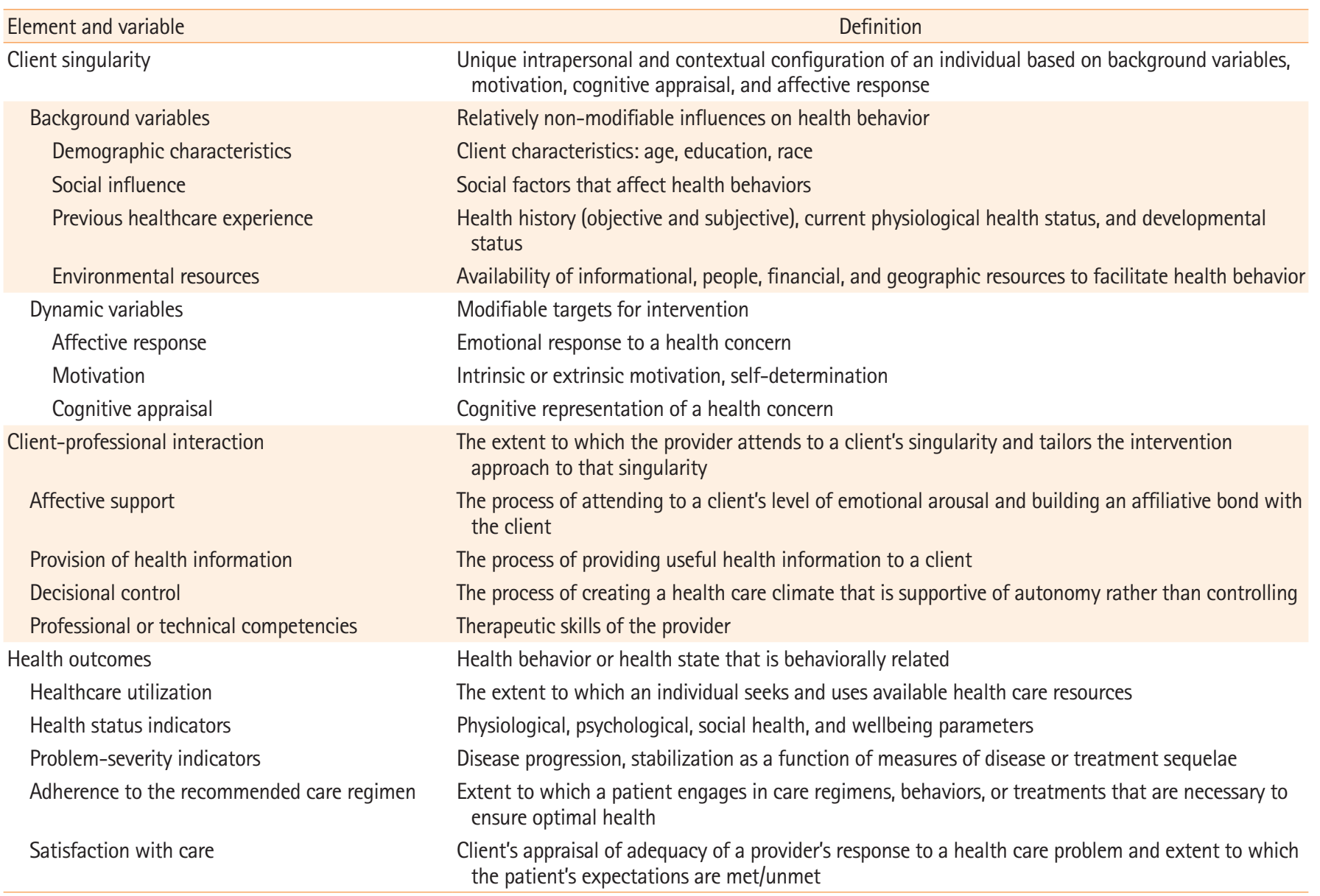


ing interventions involve emotional support, provision of health information, decisional control, and professional/technical competencies through interactions with the client [7].

Cox [15] stated that the IMCHB can be used for the client's health care based on a holistic philosophy. Therefore, it was confirmed that IMCHB contains all four concepts of the metaparadigm of nursing and views health from a holistic philosophical point of view.

\section{Content: Concepts and propositions}

The IMCHB is composed of three elements: the singularity of the client, interaction between the client and the professional, and health outcome of the client (Figure 2). It contains seven concepts regarding the client's singularity, four concepts relating to patient-professional interactions, and five concepts involving the client's health outcomes (Table 2).

An example drawn from the nine statements that correspond to the IMCHB's conceptual explanation is "the background variable of the theory includes the demographic characteristics and includes social influence, previous health care experiences, and environmental resources," [7]. Relationship statements are divided into those that express correlation and those that express causality; an example of a correlational statement is "the interrelationships between cognitive appraisal, intrinsic motivation and affective response are complicated," and an example of a causal statement is "the background variables, which include sociocultural and personal resource, directly influence the individual's cognitive appraisal" [7].

\section{Evaluation of the theory}

\section{Significance}

The significance of a theory is evaluated to check whether concepts and propositions are specified based on philosophical opinions, and whether conceptual models are presented based on philosophical opinions [10]. Cox's IMCHB is a targeted health promotion theory developed from a holistic point of view of humans and nursing, and aims to induce health behavior through interactions between experts and clients by identifying clients' background, as well as relevant cognitive and psychological factors. The IMCHB presents three main elements and sub-concepts, and shows their interactions. Cox's original formulation of the IMCHB did not specify the definition and measurement method of the concepts, but another study by Cox [16] explained each conceptual and operational definition.

\section{Internal consistency}

Internal consistency ensures that the elements of a theory, the meaning and clarity of the concepts, and terms and definitions are consistent [10]. In the 11 studies reviewed in this study, [1727] only seven experimental studies included the three major components of the IMCHB [18, 20-25, 27]. All the dynamic variables included in elements of the client's singularity were

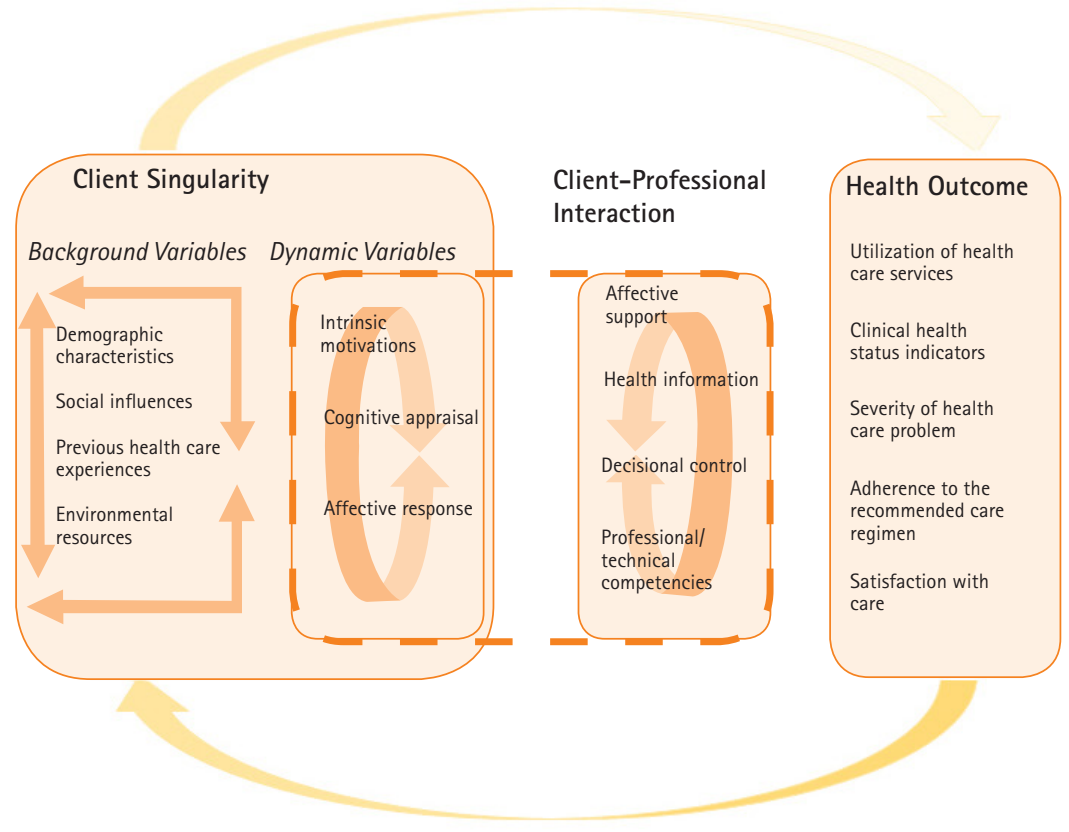

Figure 2. Interaction model of client health behavior.

Adapted from Cox et al. [16] (Oncol Nurs Forum 2003;30(5):E92-E99) with original copyright holder's permission. 
displayed in two descriptive studies $[21,26]$ and one experimental study [22]. Of the four studies $[21,22,23,25]$ that aimed to measure intrinsic motivation, that by Hanrungcharotorn et al. [21] actually measured extrinsic motivations for health behaviors through support from family, friends, and colleagues, but not intrinsic motivations for self- determinants of health behavior within the IMCHB. In seven experimental studies, the elements of client-professional interaction were included, but in two studies, the conceptual meaning of "decisional control" and "professional technical competencies" were unclear.

The relationships among the variables of client-professional interactions, such as affective support, health information, decision control, and professional/technical competencies, were also unclear.

\section{Parsimony}

The parsimony of a theory refers to whether phenomena are clearly explained using as few concepts and propositions as possible. Cox's IMCHB includes three main elements and a total of 16 sub-concepts. The relationships among the elements and among the concepts are shown in Figure 2. Among the reviewed studies, none measured all 16 sub-concepts, and only three measured affective response, motivation, and cognitive appraisal, corresponding to the dynamic variables affecting health outcomes $[21,22,26]$. In two studies $[18,22]$ that presented a theoretical framework, the interactions between client's background variables, such as demographic characteristics, social influence, and previous health care experiences, were not specified.

\section{Generality}

The generality of a theory refers to whether it is applicable to various population groups [11]. The participants of the reviewed studies were women of various groups, including minorities (immigrants or multicultural women) and low-income women $[17,19,23,24,26]$, pregnant women [22,25], postpartum women [27], women with gynecological cancer [18], women with osteoarthritis [21], and older adults [20], suggesting adequate generality.

\section{Testability}

Testability refers to whether the scientific usefulness of a theory can be empirically tested. It can be evaluated in terms of whether research methodology reflects theory, whether there are scales to measure observable concepts, and whether statistical analysis can measure its propositions [10].

In seven studies (63.6\%), nursing interventions were provided to the participants, reflecting the specificity of the nursing prescriptions presented in the IMCHB. The main health outcomes measured in the 11 studies were health status [17,22], problem-severity indicators $[18,20,21,23,25]$, adherence to the recommended care regimen [22,24-26], and satisfaction with care [27]. The reviewed studies measured concepts using different scales for each. In two studies, depression status was measured using the Center for Epidemiologic Studies Depression Scale, and pain was measured using a visual analog scale.

The studies used the following statistical methods: the chisquare test; the t-test; analysis of variance (ANOVA), analysis of covariance, and repeated-measures ANOVA; the Mann-Whitney U-test; the Kruskal-Wallis test; correlation analysis; regression analysis; and survival analysis.

\section{Empirical adequacy}

To evaluate the empirical adequacy of a theory is to analyze whether the theory is consistent with the empirical assertions derived from the research [10]. In the reviewed studies, health outcomes were confirmed according to the variables of client singularity, which are elements of the IMCHB (Table 3). Of the seven experimental studies, six studies based on the IMCHB $[18,20,22-25]$ provided interventions that considered the clients' background variables and improved health outcomes through client-professional interactions. Cox [16] claimed that previous health outcomes could provide feedback regarding a client's background variables. In Ackerson's study [17], previous experiences of Pap smears in minority women were an important factor in receiving regular cervical cancer screening tests. In addition, affective response, intrinsic motivation, and cognitive appraisal—as dynamic variables of the client-were partially supported, with positive effects on health outcomes.

\section{Pragmatic adequacy}

A theory's pragmatic adequacy is evaluated using the criteria of (a) the education and training required before utilization in nursing practice, (b) the theory's application in real nursing practice, (c) the feasibility of implementing theoretical activities, and (d) the professional's legal capacity to implement the activities [10]. In the reviewed studies, the IMCHB was applied to women's health promotion in a variety of nursing practices, such as cervical cancer screening, postpartum management, breastfeeding, and physical activity enhancement. These were studies conducted in real-world clinical settings and helped solve the participants' health problems. In the study of An et al. [18], when caring for gynecological cancer patients, nurses encouraged physical activity, imparted nutritional education to the participants, and stimulated an affective response. Based on 


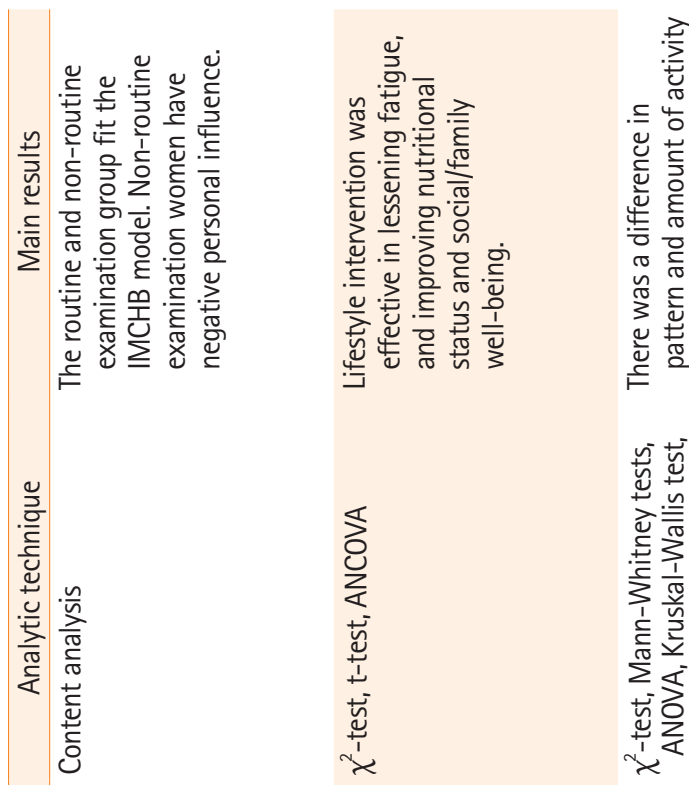

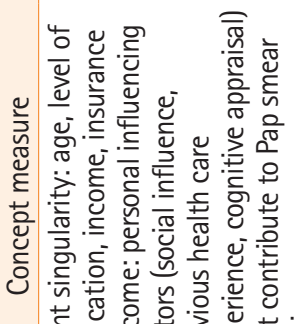

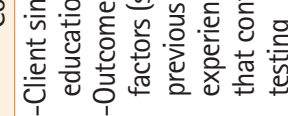

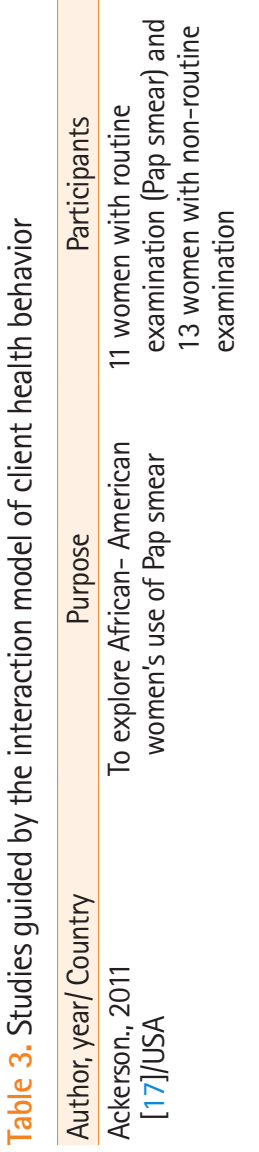

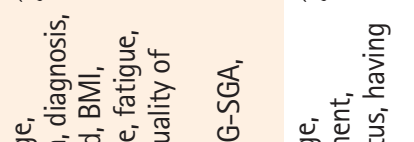

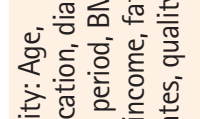

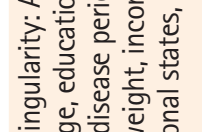

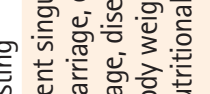

岕

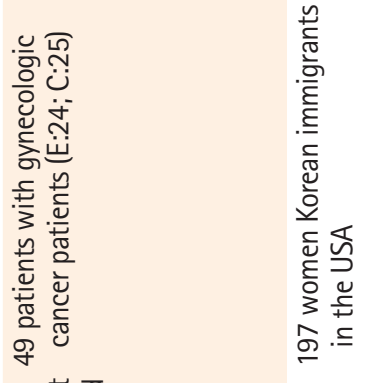

苍蒂焉

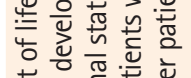

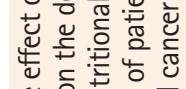

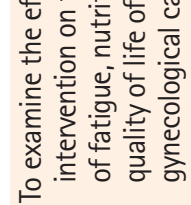
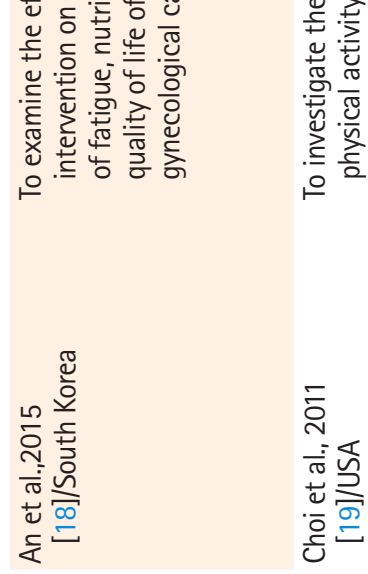

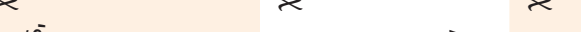

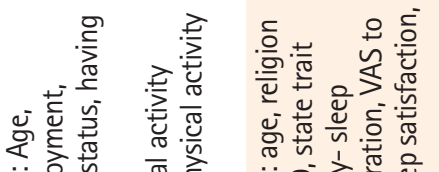

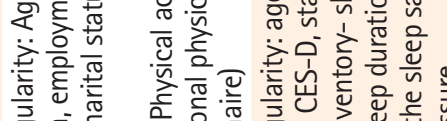

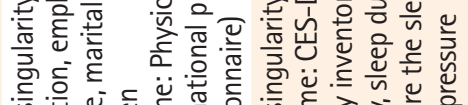
它

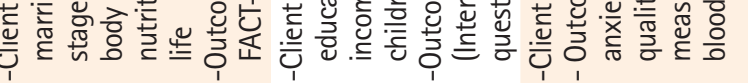
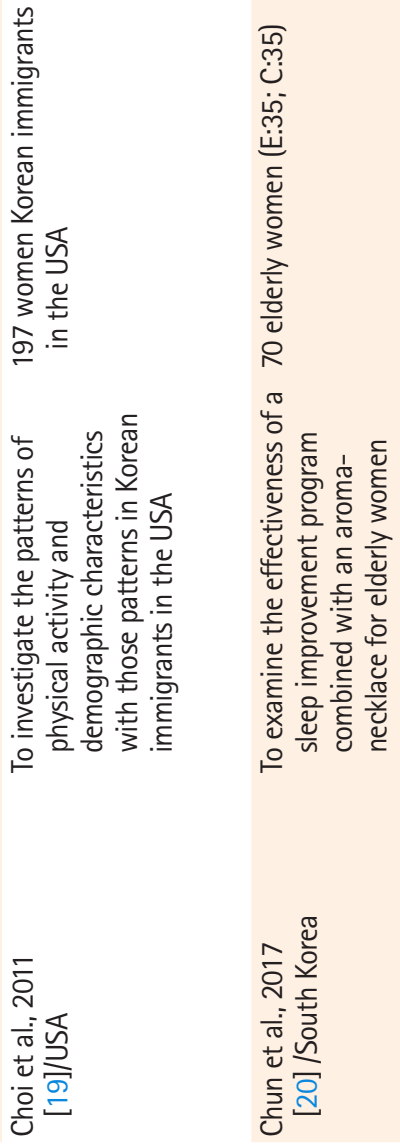

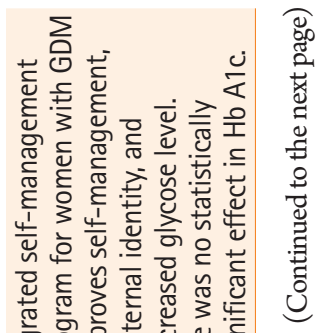

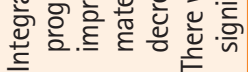
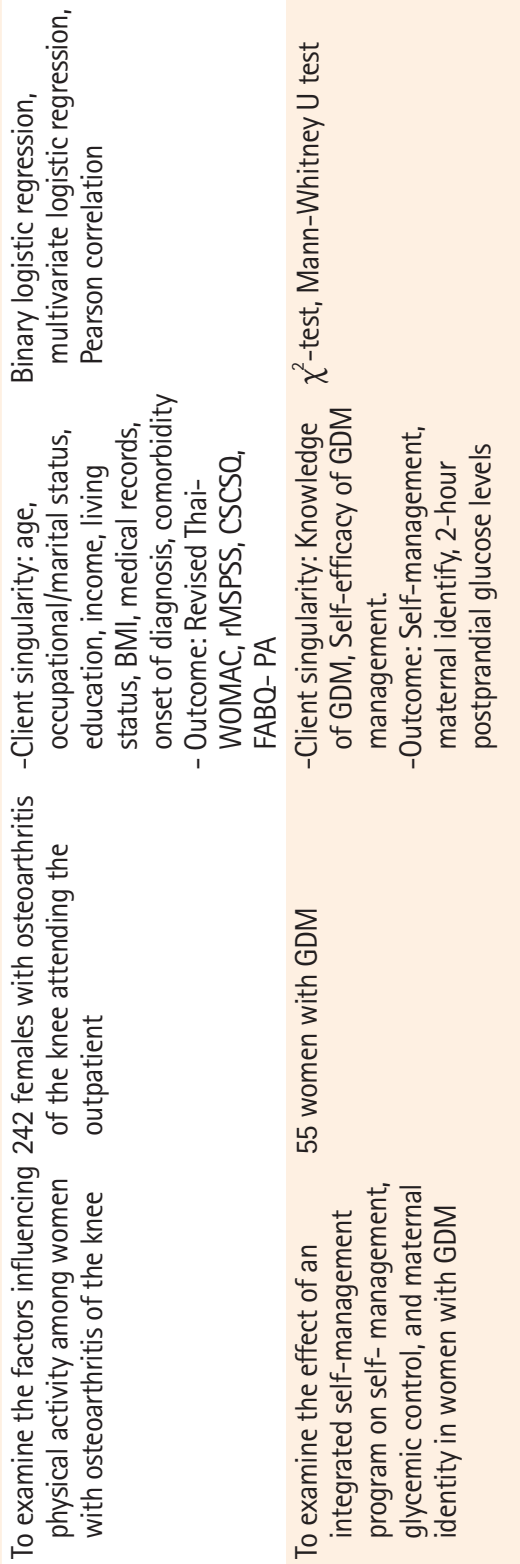

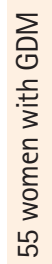

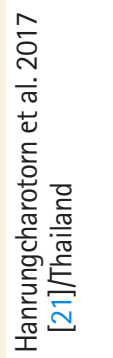

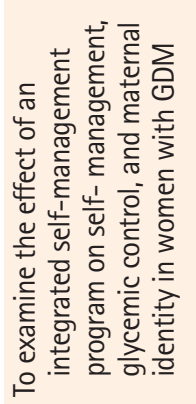

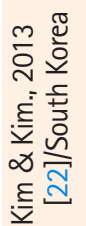



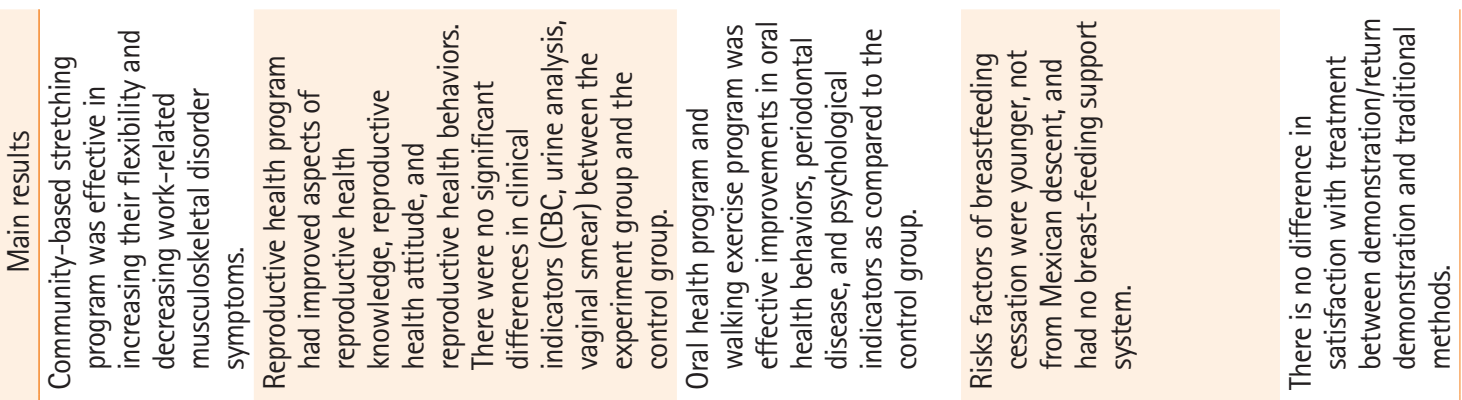

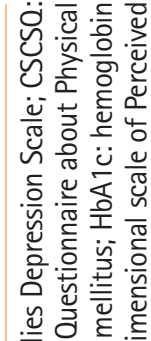

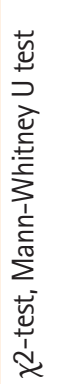

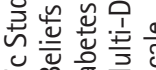
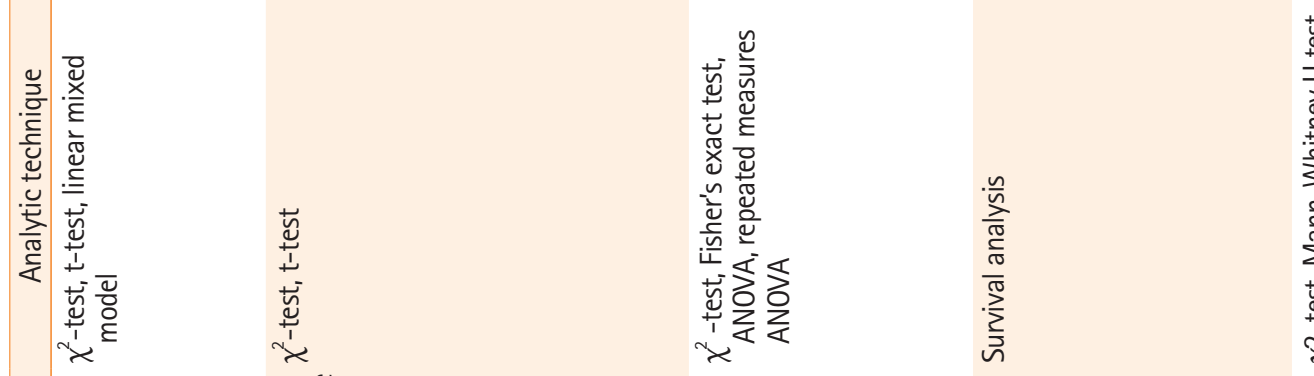

는
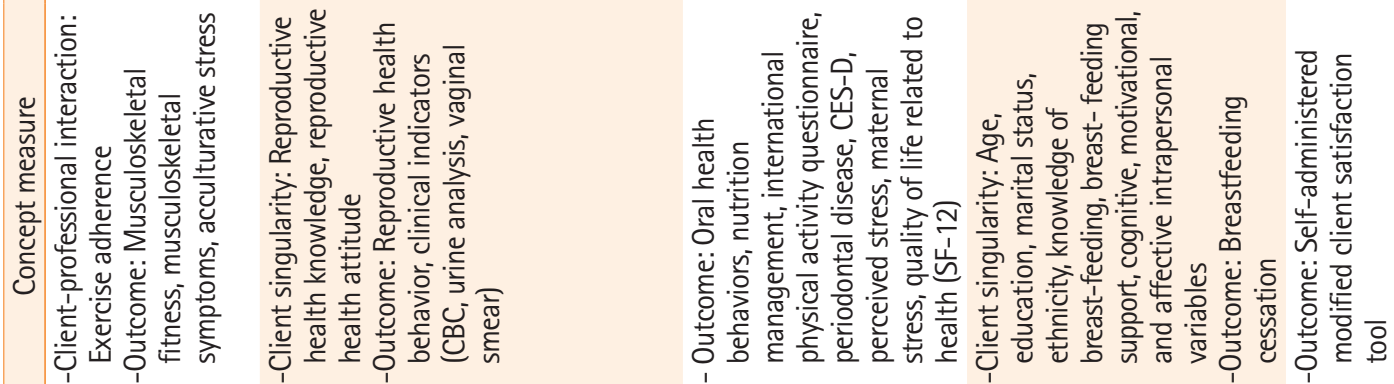

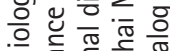

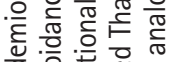

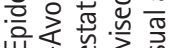

ᄒᄒ $\frac{1}{\pi}$ o d

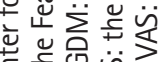

U⿺辶寸

oิ들 $\sum_{i}$

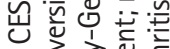

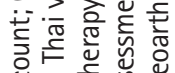

Uे

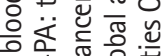

엉 은 는

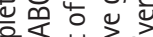

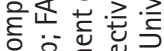

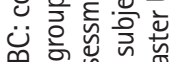

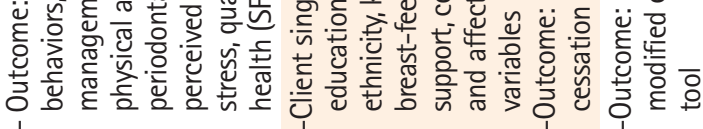

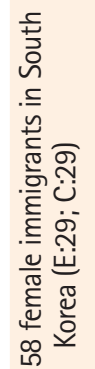

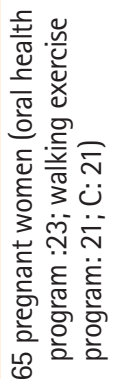

4

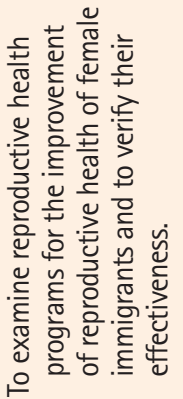

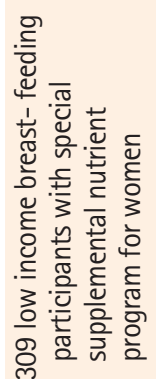

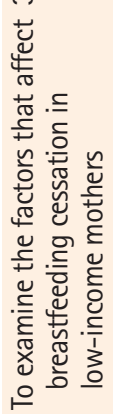

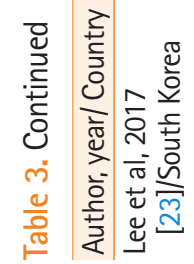
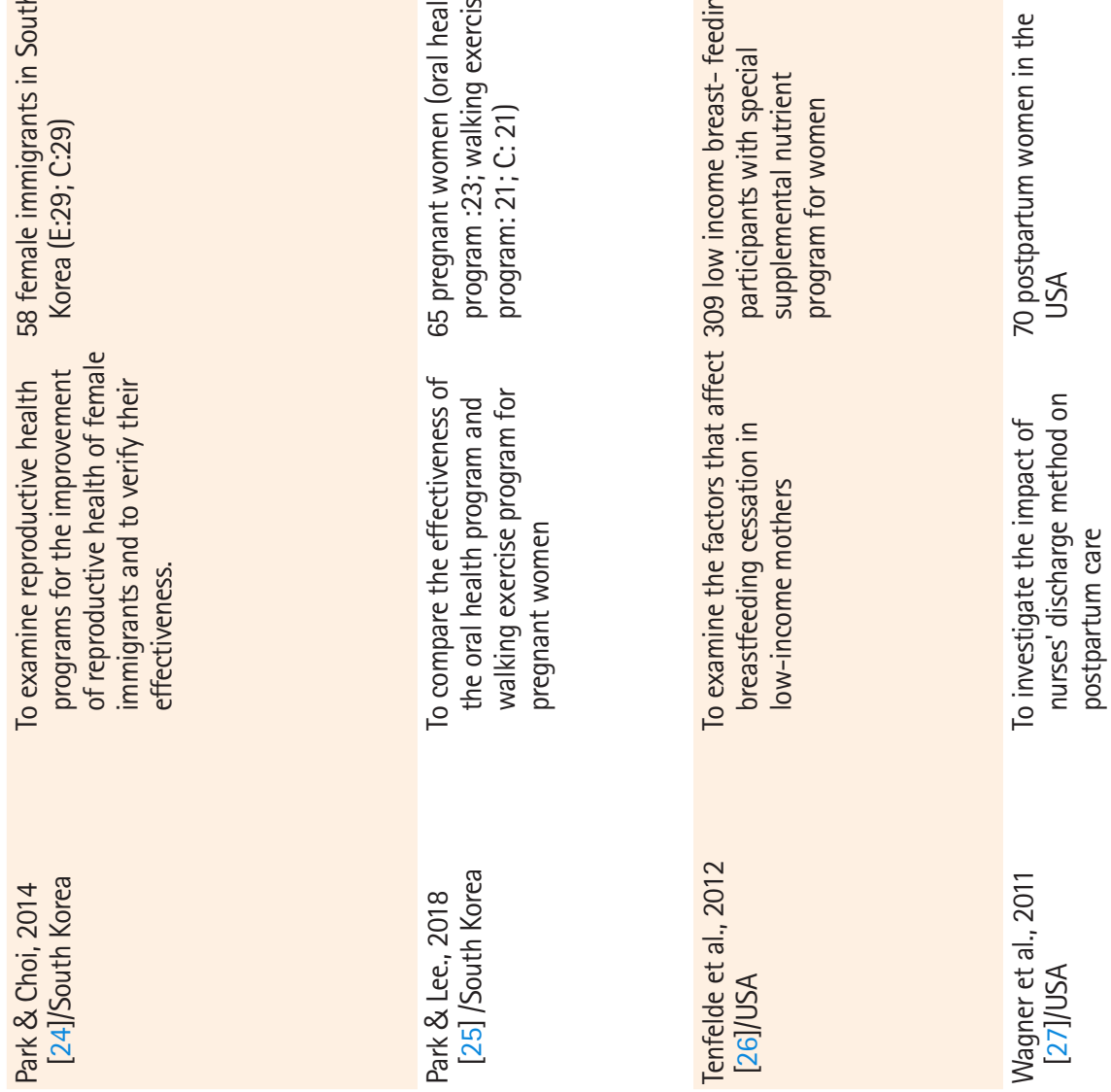
these results, it can be concluded that Cox's IMCHB has excellent pragmatic adequacy.

\section{Discussion}

In this study, the structural and functional elements of Cox's IMCHB were confirmed through a review of articles on women's health and the application of the IMCHB, based on the criteria of Fawcett [10] and Chinn and Kramer [11]. Moreover, based on the results of the evaluation, this study aimed to present certain directions to be considered when providing and evaluating nursing interventions that apply the IMCHB in future women's health research.

First published in 1982, Cox's IMCHB [7] presents a comprehensive framework in nursing practice, education, and research, including the metaparadigm of nursing. It also emphasizes the philosophical assumptions of nursing from a holistic point of view [28], and underscores the need to assess health from a multidimensional perspective, taking into account the physical, cognitive, and psychological aspects of clients. The IMCHB is special in acknowledging the individual's singular identity and the right to select health behaviors for their health promotion. It also presents a nursing metaparadigm, furnishes a theoretical framework for evaluating nursing intervention, provides professional/practical health promotion guidelines for nurses, and has contributed to the expansion of knowledge in nursing research. In addition, upon reviewing 11 studies, it was found that the IMCHB has been used as a health promotion intervention strategy for various populations of women and has led to useful results in nursing practice; therefore, it is a suitable theory for experimental and clinical research.

The meanings of the sub-concepts of the three elements of the IMCHB were not unified in each study and were unclear, so internal consistency was somewhat insufficient in the studies included in this review. Cox's IMCHB includes many concepts and complicated relationships, and therefore lacks clarity and parsimony.

The IMCHB was developed with the goal of devising the best interventions possible by focusing on the interrelationship between clients and nurses [7]. Cox [16] claimed that the IMCHB can guide explanatory and intervention studies for health behavior. This review confirmed that the IMCHB has been applied to suggest directions for nursing interventions and has been helpful for improving health behavior, with positive effects on health outcomes.

After developing the IMCHB, Cox [15] developed the health self-determinism index to systematically measure various intrin- sic motivations for health behavior. However, the health self-determinism index was not used in the reviewed literature; on the contrary, in one study, intrinsic motivation was incorrectly measured in terms of extrinsic motivation. This indicates the need for a clear analysis and understanding of the theory and its concepts before applying it to research. No studies measured all five health outcomes or the utilization of healthcare services.

The development of the IMCHB started with the aim of developing a theory of health behavior that can be practically used from the nursing perspective [7]. Accordingly, this review confirmed that IMCHB has clearly guided practice and research to provide appropriate nursing for patients. Also, as a high-level prescribing theory, the IMCHB can be helpful in nursing research and practice to develop further knowledge in the field of nursing [29].

In summary, this review revealed that the IMCHB has significance, generality, testability, empirical adequacy, and pragmatic adequacy for nursing practice and research. However, the lack of clear conceptual definitions and the complex relationships among concepts result in a lack of internal consistency and parsimony. As such, the IMCHB is a suitable theory for guiding practice and research in women's health promotion and the development and application of interventions. Future research can build on this middle-range theory in women's health research and practice.

\section{ORCID}

Youlim Kim, https://orcid.org/0000-0002-4477-2489

Hyeonkyeong Lee, https://orcid.org/0000-0001-9558-7737

Gi Wook Ryu, https://orcid.org/0000-0002-4533-7788

\section{Authors' contributions}

Design: Kim Y, Lee H, Ryu GW; Literature review: Kim Y, Ryu GW; Theory analysis: Kim Y, Ryu GW; Writing-original draft: Kim Y, Ryu GW; Writing-review \& editing: Kim Y, Lee H, Ryu GW.

\section{Conflict of interest}

The authors declared no conflict of interest.

\section{Funding}

None. 


\section{Data availability}

Please contact the corresponding author for data availability.

\section{Acknowledgments}

The authors would like to thank the Oncology Nursing Society for allowing us to use the figure presenting Cox's theory.

\section{References}

1. Lee MS. Global health promotion efforts for achieving the UN 2030 agenda for Sustainable Development. Korean J Health Edu Promot. 2018;35(4):1-18. https://doi.org/10. 14367/kjhep.2018.35.4.1

2. Lee YJ, Kim SY, Kang SY, Kang YJ, Jin L, Jung HY, et al. Trend analysis of research articles published in the Korean journal of women health nursing from 2013 to 2017. Korean J Women Health Nurs. 2018;24(1):90-103. https://doi.org/10.4069/ kjwhn.2018.24.1.90

3. Lee KH, Han YR, Bai JI, Jeong GH. Concept analysis on woman health. J Korean Acad Women Health Nurs. 1995; 1(2):222-233.

4. Han NK, Kim S, Kim MS, Kim JG, Kim CH, Choi SH. Trend analysis of research in the Korean journal of adult nursing for 5 years (2010 2014): focused on usage of nursing theories. Korean J Adult Nurs. 2015;27(5):527-536. https://doi. org/10.7475/kjan.2015.27.5.527

5. Lee SW, Kim JH, Lee BS, Jung MS, Yoo M, Lee DS, et al. Understanding of nursing theory. Paju: Sumunsa; 2017. $491 \mathrm{p}$.

6. Cho BH. Health promotion concept and theory. Asian Oncology Nursing. 2008; 8(1Sup), 9-20

7. Cox CL. An interaction model of client health behavior: theoretical prescription for nursing. ANS Adv Nurs Sci. 1982;5(1):41-56. https://doi.org/10.1097/00012272-1982 10000-00007

8. Im EO. The current status of theory evaluation in nursing. J Adv Nurs. 2015;71(10):2268-2278. https://doi.org/10.1111/ jan. 12698

9. Carter KF, Kulbok PA. Evaluation of the Interaction Model of Client Health Behavior through the first decade of research. ANS Adv Nurs Sci. 1995;18(1):62-73. https://doi.org/ 10.1097/00012272-199509000-00007

10. Fawcett J. Criteria for evaluation of theory. Nurs Sci Q. 2005;18(2):131-135. https://doi.org/10.1177/089431 8405274823

11. Chinn P, Kramer M. Integrated theory and knowledge devel- opment in nursing. 8th ed. St. Louis, MO: Elsevier Mosby; 2011.272 p.

12. Walker L, Avant K. Strategies for theory construction in nursing. 4th ed. Upper Saddle River, NJ: Pearson/Prentice Hall; 2005. $227 \mathrm{p}$

13. Yi M, Lee SW, Kim KJ, Kim MG, Kim JH, Lee KH, et al. A review study on the strategies for concept analysis. J Korean Acad Nurs. 2006;36(3):493-502. https://doi.org/10.4040/ jkan.2006.36.3.493

14. Cox CL, Roghmann KJ. Empirical test of the interaction model of client health behavior. Res Nurs Health. 1984; 7(4):275-285. https://doi.org/10.1002/nur.4770070406

15. Cox CL. The Health Self-Determinism Index. Nurs Res. 1985;34(3):177-183.

16. Cox CL. Online exclusive: a model of health behavior to guide studies of childhood cancer survivors. Oncol Nurs Forum. 2003;30(5):E92-E99. https://doi.org/10.1188/03.ONF. E92-E99

17. Ackerson K. Interactive model of client health behavior and cervical cancer screening of African-American women. Public Health Nurs. 2011;28(3):271-280. https://doi.org/10.1111/ j.1525-1446.2010.00901.x

18. An H, Nho JH, Yoo S, Kim H, Nho M, Yoo H. Effects of lifestyle intervention on fatigue, nutritional status and quality of life in patients with gynecologic cancer. J Korean Acad Nurs. 2015;45(6):812-822. https://doi.org/10.4040/jkan.2015. 45.6 .812

19. Choi J, Wilbur J, Kim MJ. Patterns of leisure time and non-leisure time physical activity of Korean immigrant women. Health Care Women Int. 2011;32(2):140-153. https://doi. org/10.1080/07399332.2010.508131

20. Chun N, Kim M, Noh GO. Effects of a sleep improvement program combined with aroma-necklace on sleep, depression, anxiety and blood pressure in elderly women. J Korean Acad Nurs. 2017;47(5):651-662. https://doi.org/10.4040/ jkan.2017.47.5.651

21. Hanrungcharotorn U, Pinyopasakul W, Pongthavornkamol K, Dajpratham P, Beeber AS. Factors influencing physical activity among women with osteoarthritis of the knee. Pac Rim Int J Nurs Res. 2017;21(1):5-17.

22. Kim HS, Kim S. Effects of an integrated self-management program on self-management, glycemic, control, and maternal identity in women with gestational diabetes mellitus. J Korean Acad Nurs. 2013;43(1):69-80. https://doi.org/10. 4040/jkan.2013.43.1.69

23. Lee H, Chae D, Cho S, Kim J, Yoo R. Influence of a community-based stretching intervention on the health outcomes 
among Korean-Chinese female migrant workers in South Korea: a randomized prospective trial. Jpn J Nurs Sci. 2017;14(4):277-287. https://doi.org/10.1111/jjns.12156

24. Park MN, Choi SY. Development of reproductive health program and identification of effect for married women immigrants. J Korean Acad Nurs. 2014;44(3):248-258. https://doi. org/10.4040/jkan.2014.44.3.248

25. Park HJ, Lee H. Comparison of effects of oral health program and walking exercise program on health outcomes for pregnant women. J Korean Acad Nurs. 2018;48(5):506-520. https://doi.org/10.4040/jkan.2018.48.5.506

26. Tenfelde SM, Finnegan L, Miller AM, Hill PD. Risk of breastfeeding cessation among low-income women, infants, and children: a discrete time survival analysis. Nurs Res.
2012;61(2):86-95. https://doi.org/10.1097/NNR.0b013e$3182456 \mathrm{~b} 0 \mathrm{a}$

27. Wagner DL, Bear M, Davidson NS. Measuring patient satisfaction with postpartum teaching methods used by nurses within the interaction model of client health behavior. Res Theory Nurs Pract. 2011;25(3):176-190. https://doi.org/ 10.1891/1541-6577.25.3.176

28. Kim HS. The nature of theoretical thinking in nursing. 3th ed. New York: Springer; 2010. 335 p.

29. Mathews SK, Secrest J, Muirhead L. The Interaction Model of Client Health Behavior: a model for advanced practice nurses. J Am Acad Nurse Pract. 2008;20(8):415-422. https://doi. org/10.1111/j.1745-7599.2008.00343.x 